\title{
Repercusiones sociales de la entrada de España en la CEE
}

\author{
M. ${ }^{a}$ Pilar Borderías URibeondo
}

\section{INTRODUCCION}

Generalmente, cuando nos referimos a la CEE y a las repercusiones que tendrá nuestra integración en esta organización, el pensamiento se va directamente hacia aspectos de orden económico, dejando de lado los aspectos sociales. Lo cierto es que debemos tener muy en cuenta que el sector social juega también un papel fundamental, puesto que, aunque inicialmente la CEE se haya basado en unos intereses económicos, podríamos considerar que éstos son un nexo de atracción inicial, ya que en el fondo de la Comunidad subyace un deseo más amplio, consistente en establecer intereses comunes a todos los niveles para llegar a configurar una comunidad integrada y solidaria que constituya una gran potencia política, social y económica en el marco mundial. Naturalmente cabe esperar que este hecho tarde cierto tiempo en conseguirse, sobre todo si tenemos en cuenta que se intenta unir paises que han estado históricamente no sólo divididos sino también enfrentados.

El interés que tienen los distintos aspectos sociales se pone de manifiesto en la propia actuación de la CEE, a través de su política social y a través de las diversas publicaciones y comités creados al efecto. La actuación de la Comunidad se eníoca hacia diversas preocupaciones e intereses cotidianos de los europeos como pueden ser: los problemas laborales, la salud, la cultura, la marginación social... etc., y se hace referencia constantemente a la "europa de los ciudadanos" con una tendencia a realizar una labor psicológica, centrada sobre todo en la juventud, ya que a ésta se la 
considera como el porvenir de Europa. Su labor en este sentido trata de desarrollar los intercambios en trabajo, estudios, idiomas, vacaciones, cultura... etc.

Evidentemente, esta labor de concienciación del ciudadano está en la base de que pueda llegar a considerarse algún día como un ciudadano europeo. Para medir la evolución de este sentir suelen publicarse periódicamente encuestas (eurobarómetro) en las que se tantea la opinión popular. En una de estas encuestas realizadas en nuestro pais en 1981, se puso de manifiesto que entre un 50 por 100 y un 60 por 100 de las personas encuestadas:

- estaban a favor de los esfuerzos por lograr la unificación europea;

- estaban interesados por los problemas de la CEE;

- se declaraban partidarios de la adhesión.

En cambio, entre lo que los españoles esperaban de la CEE no aparecian las cuestiones sociales.

No obstante, a pesar del interés centrado en la politica social, los logros comunitarios en este campo no han llegado a un alto nivel de satisfacción, pudiéndose afirmar que éstos están más avanzados a un nivel dialéctico que a un nivel legislativo, quedando en un punto intermedio el nivel financiero, que por su parte es un importante elemento de juicio para valorar el interés puesto en los aspectos sociales.

Si analizamos el reparto de los presupuestos comunitarios, lo primero que nos llama la atención es el desequilibrio tan grande que existe entre el dinero destinado a la política agraria, que es la que se considera como más integrada, y el resto de las políticas comunitarias. Según los datos correspondientes a 1983, al capítulo social se le dedicó el 5,7 por 100 de los gastos, de los cuales las 9/10 partes se efectuaron a través del Fondo social europeo, que es el medio de acción más concreto de la Comunidad en el campo social.

Desde 1984 , el 75 por 100 de los fondos dedicados a la política social se emplean en financiar medidas relativas a la politica de mercado de trabajo de los jóvenes. Concretamente en 1986 se destinaron 1.920 millones de 
ECU sólo para ayuda del desempleo juvenil. En cuanto a la distribución por países es de resaltar que se destinó a España un 13,9 por 100 (355,8 millones ECU) y a Portugal un 8,8 por 100 (223,8 millones ECU), es decir, que casi $1 / 4$ parte del presupuesto de acción social se dedicó a los dos nuevos países comunitarios, lo cual es ya un indicativo del interés de la CEE por mejorar nuestros problemas sociales.

En un principio las regiones españolas que se verán más favorecidas son:Andalucia, Canarias, Castilla-León, Castilla-La Mancha, Extremadura, Galicia, Murcia, Ceuta y Melilla.

\section{LINEAS GENERALES DE LA POLITICA SOCIAL COMUNITARIA}

El conocimiento de la política social es importante para poder plantearnos las repercusiones que tendrá nuestra adhesión a la CEE.

Podemos considerar como un momento clave en el impulso de esta política comunitaria la crisis económica de los años 70 , llegándose a fijar en 1974 una serie de objetivos relacionados con el tema laboral. Prácticamente, hasta 1973 el único logro conseguido estaba en relación con la libertad de circulación y la igualdad de trato para los trabajadores emigrantes y sus familias. De forma que el programa de acción social del año 1973 puede considerarse como la primera manifestación de la responsabilidad comunitaria en la promoción del progreso social y en la intervención activa para conseguir mejorar las condiciones de vida y de trabajo.

En realidad, los años siguientes a este programa, en los que se agudizaron la crisis económica y las tensiones políticas, no fueron años propicios para la realización de unas mejoras acusadas en el campo de la política social, aunque parece ser que fueron precisamente el propio incremento de las tasas de paro y el descenso en el nivel y en la calidad de vida los que sirvieron de impulso para el progreso de una acción social posterior.

A pesar de estos problemas cabe destacar el desarrollo de una legislación vinculante, expresada a través de Directivas basadas en los artículos 100 y 235 del tratado de Roma. En los últimos años se ha planteado además la necesidad de una política social más dinámica, basada en el diálogo social y en la búsqueda del consenso. No obstante, hay que considerar que todavia 
existe poca integración política entre los países comunitarios en este aspecto, de forma que los aspectos sociales comunitarizados son pocos si los comparamos con la gran cantidad de normas vigentes en los distintos Estados Miembros, en los que además se tratan de forma diferente los problemas del sector social.

La situación en que se encuentra actualmente la política social varía según distintos criterios, aunque una mayoría coincide en afirmar que el progreso ha sido escaso y que, en general, es todavia una de las políticas menos desarrolladas en el ámbito de la CEE.

En líneas generales, la política social se ha propuesto los siguientes objetivos:

POLITICA SOCIAL DE LA CEE: ACCIONES PRIORITARIAS EN LA ACTUALIDAD

LUCHA CONTRA EL PARO

PROBLEMAS DE LA JUVENTUD
- Acciones de formación y reconversión profesional.

- Reducir desequilibrios entre la oferta y la demanda de trabajo (SEDOC).

- Estimular un crecimiento económico más generador de empleo/Relanzamiento de la inversión.

- Esfuerzos especiales en los sectores más afectados (jóvenes, mujeres...)

- Empleo: Financiación a las empresas que creen empleo juvenil.

Apoyo a los jóvenes creadores de empresas. Promoción de inserción profesional a los jóvenes menos favorecidos.

- Educación: Mejorar la preparación para la actividad profesional.

Cooperación europea entre Univ. y centros de Estud. Superiores. Movilidad estudiantil: intercambios entre Universidades.

Créditos en favor de la educación y la cultura.

- Formación profesional: Adaptación a las necesidades económicas y sociales actuales y futuras. 
MEJORA DE LAS CONDICIONES DE TRABAJO
PROBLEMAS DE LOS

EMIGRANTES

LUCHA CONTRA LA POBREZA

IGUALDAD DE LA MUJER
- Objetivo: "Contribuir tanto al bienestar de los trabajadores como al proceso de la productividad".

- Mayor participación de los trabajadores en las decisiones de la empresa.

- Seguridad y salud en el lugar de trabajo.

- Protección de los derechos del trabajador.

- Mejor reparto del trabajo.

- Conseguir una mayor cualificación profesional de los trabajadores.

- Libre circulación de trabajadores.

- Mejorar la situación de los emigrantes por medio de:

Coordinación de sistemas nacionales de seguridad social. Derechos sindicales de los emigrantes.

Conservación de la cultura del país de origen en la enseñanza.

Mejora de las condiciones de alojamiento.

- Mejora de las condiciones de vida.

- Ayudas especiales a las regiones más pobres o más afectadas por el paro.

- Ayudas concretas que afectan a: marginados; ancianos; refugiados y migrantes de retorno; parados de larga duración; familias monoparentales.

- Eliminar las causas específicas del elevado porcentaje de paro.

- Igualdad de remuneración entre hombres y mujeres.

- Igualdad de trato en el acceso al empleo; formación y promoción profesional; condiciones de trabajo; régimen legal de seguridad social.

- Estimular la participación de la mujer en los sectores profesionales en que estaba infrarrepresentada.

- Minusválidos: Promoción de empleo de minusválidos. Mayor participación en la vida social. Mejorar su ambiente y favorecer su movi lidad.

- Ancianos.

- Margiriados en general. 


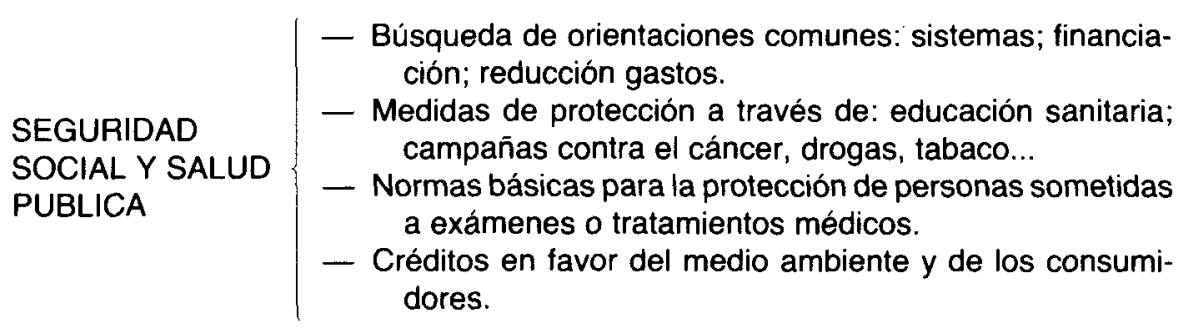

Ahora bien, aunque estos puntos constituyen la política general de la $\mathrm{CEE}$, tenemos que tener siempre presente la cantidad de intereses contrapuestos entre los diversos países que constituyen la Comunidad, los cuales provocan un conflicto generalizado en cuanto a la homologación de las respectivas políticas sociales. En nuestro caso, por ejemplo, se asegura que en el capítulo social se mantuvieron fuertes discusiones durante el proceso de negociación entre España y la CEE, llegándose a opinar incluso que las posturas comunitaria y española en materia social fueron más distantes que en otros campos.

Este hecho no debe extrañarnos puesto que, la mayor parte de las veces, las mejoras sociales conllevan conflictos económicos, lo que explica la cautela con que se lleva este aspecto comunitario. A título orientativo no hay más que observar las diferenciaciones que se hacen entre "normas" $y$ "pautas de comportamiento", es decir, que en unos casos existe una obligatoriedad de cumplimiento, pero en otros es solamente un consejo que se da. Por otro lado hay todavia un gran número de aspectos que están en fase de proyecto como norma a seguir.

Ante esta circunstancia hay quien opina que la importancia de la política social de la CEE no radica tanto en las normas que pudieran tener una vigencia general, sino en lo que pudiéramos llamar "su efecto ejemplificador», en el sentido en que las normas aplicables en alguno de los Estados tienden a ser imitadas en los restantes, ya que afectan a la población de una forma directa. En este sentido, J. A. PAREDO (1984) opina que «... la incidencia en nuestro pais será reducida desde un punto de vista formal, por las características de la legislación social española que parece estar en línea con los principios vigentes en la mayor parte de los paises comunitarios, en cambio parece ser que afectará más a modificaciones en la mentalidad y en la conducta de las personas frente a determinadas circunstancias». 
No obstante, será necesario cambiar diversas normativas para adaptarnos plenamente, como por ejemplo en las referentes a: regulación del trabajo de los extranjeros en España; equiparaciones en distintos órdenes (fiscal, sanitario, de transporte...); limitaciones de las causas de expulsión de extranjeros; normas reguladoras de la Seguridad Social; equivalencias entre los sistemas y niveles educativos..., etc.

A la hora de considerar las repercusiones de nuestra entrada en la CEE hay que tener también en cuenta los aspectos subjetivos, como son los planteamientos o puntos de partida de quien vaya a dar su opinión sobre dichas repercusiones, puesto que esa opinión irá en función del mayor o menor cumplimiento de los objetivos propuestos: asi, desde las perspectivas del momento en que se solicitó la adhesión, no queda clara una futura respuesta positiva a todos los niveles por los cambios y las crisis habidas en los años posteriores a dicha solicitud (en ese momento había dos objetivos claros: por un lado la consolidación de la democracia y la reafirmación de establecimiento de las libertades públicas, al considerarse que la participación en la Comunidad nos daría un carácter europeo; y por otro lado estaba la imagen europea de bienestar y de elevado nivel de vida, lo cual representaba un ideal para el ciudadano medio español).

Analizando las repercusiones con otras perspectivas y visto desde el momento actual, podremos sacar diversas conclusiones como iremos viendo a lo largo de esta exposición.

Los aspectos jurídicos hasta aquí resumidos, representan una base objetiva de partida para identificar aspectos que repercutirán necesariamente sobre nuestras normas sociales; y a continuación analizaremos la composición y la estructura de la población y sus problemas demográficos, los cuales están en la base de esa política social comunitaria.

El desarrollo de esta ponencia se centra fundamentalmente en plantear una serie de cuestiones en torno a las características poblacionales de la Europa de los Doce, de forma que, comparándolas con las normativas de la política social comunitaria, pueda sacar cada cual sus propias conclusiones sobre la repercusión que tendrá la adhesión de España a la CEE, puesto que en el momento actual la mayor parte de las opiniones emitidas son meras hipótesis, al no haber transcurrido un tiempo suficiente para constatar hechos precisos; al tiempo que también hay que tener en cuenta que todavía estamos en el periodo transitorio de siete años, que finalizará en 1992 y hasta entonces no se harán efectivas muchas de las cuestiones pactadas. 


\section{COMPOSICION DE LA POBLACION DE LA CEE}

Analizando la población de la Europa de los Doce en su conjunto, se hace patente la importancia de la unión de todos los países comunitarios si consideramos que llegan a constituir la tercera potencia demográfica mundial, detrás de China e India, quedando por encima de EE.UU. En ella se reúne el 7 por 100 de la población mundial, mientras que su superficie no llega al 2 por 100 de la superficie terrestre.

Socio-económicamente podemos traducir este hecho en que en el momento actual somos la tercera poiencia mundial en cuanto a volumen de mano de obra y en cuanto a volumen de consumidores, pero hay que puntualizar "en el momento actual», puesto que la población de la CEE presenta un crecimiento natural más lento que el conjunto mundial, hasta el punto de que según algunos cálculos, en el año 2050 la población comunitaria representará tan sólo el 2 o el 3 por 100 de la población mundial.

Así, tenemos que mientras que hay países (generalmente los subdesarrollados) que presentan un crecimiento natural muy elevado, entre el 3 y el 4 por 100, dentro de la CEE los países tienen un crecimiento que no supera de media al 1 por 100 anual. El resultado de ello es que se está caminando hacia un envejecimiento de la población, como se deriva del porcentaje relativamente bajo de jóvenes y la elevada proporción de mayores de sesenta y cinco años. Así, entre 1970 y 1980 los porcentajes de menores de quince años decrecieron del 24,3 al 22 por 100 del total de la población, y el porcentaje de mayores de sesenta y cinco años ascendió del 12,3 al 13,9 por 100 .

No obstante, analizados individualmente los distintos países que componen la CEE, éstos presentan entre sí una gran diversidad poblacional a todos los niveles: total de población, densidad, crecimiento... etc., lo cual impide obtener una visión real de cada uno de ellos a través de las medias o de las generalizaciones. (Cuadros I y II). 
CUADRO I. DINAMICA DEMOGRAFICA

\begin{tabular}{|c|c|c|c|c|c|c|c|c|c|c|}
\hline \multirow{3}{*}{ PAISES } & \multicolumn{6}{|c|}{ MOVIMIENTO NATURAL \% } & \multirow{2}{*}{\multicolumn{2}{|c|}{$\begin{array}{l}\text { MOV. MIGRAT } \\
\\
\text { T. SALDO \% } \\
\text { NET. MIGRAT }\end{array}$}} & \multirow{2}{*}{\multicolumn{2}{|c|}{$\begin{array}{c}\begin{array}{c}\text { CRECIM. } \\
\text { TOTAL }\end{array} \\
\begin{array}{c}\text { T. CRECIM. \% } \\
\text { TOTAL }\end{array}\end{array}$}} \\
\hline & \multicolumn{2}{|c|}{$\begin{array}{l}\text { TASA DE } \\
\text { NATALIDAD }\end{array}$} & \multicolumn{2}{|c|}{$\begin{array}{c}\text { TASA DE } \\
\text { MORTALID. }\end{array}$} & \multicolumn{2}{|c|}{$\begin{array}{l}\text { T. CRECIM. } \\
\text { NATURAL }\end{array}$} & & & & \\
\hline & 1970 & 1984 & 1970 & 1984 & 1970 & 1984 & 1970 & 1984 & 1970 & 1984 \\
\hline Bélgica & 14,8 & 11,8 & 12,3 & 11,2 & 2,3 & 0,5 & 0,4 & 0,0 & 2,7 & 0,5 \\
\hline P. Bajos & 18,3 & 12,1 & 8,4 & 8,3 & 9,9 & 3,8 & 2,6 & 0,6 & 12,4 & 4,1 \\
\hline Luxemburgo & 13,0 & 11,5 & 12,2 & 11,1 & 0,8 & 0,3 & 3,1 & 0,3 & 3,8 & $-0,1$ \\
\hline Francia & 16,8 & 13,8 & 10,7 & 9,9 & 6,1 & 4,0 & 3,5 & 0,3 & 9,6 & 4,3 \\
\hline Italia & 16,8 & 10,3 & 9,6 & 9,3 & 7,2 & 1,1 & $-2,2$ & 1,6 & 5,1 & 2,7 \\
\hline RFA & 13,4 & 9,5 & 12,1 & 11,3 & 1,3 & $-1,8$ & 9,2 & $-2,4$ & 10,5 & $-4,2$ \\
\hline R. Unido & 16,2 & 12,9 & 11,8 & 11,4 & 4,5 & 1,5 & $-0,5$ & 0,9 & 4,0 & 2,4 \\
\hline Irlanda & 21,8 & 18,2 & 11,4 & 9,1 & 10,4 & 9,1 & $-1,2$ & 3,6 & 9,3 & 5,5 \\
\hline Dinamarca & 14,4 & 10,1 & 9,8 & 11,2 & 4,6 & $-1,0$ & 2,4 & 0,8 & 6,9 & $-0,3$ \\
\hline Grecia & 16,5 & 12,7 & 8,4 & 8,9 & 8,1 & 3,8 & $-5,3$ & 1,1 & 2,8 & 4,8 \\
\hline España & 19,6 & 12,5 & 8,3 & 7,7 & 11,3 & 4,7 & $-0,8$ & 0,8 & 10,5 & 5,6 \\
\hline Portugal & 20,0 & 14,2 & 10,3 & 9,6 & 9,7 & 4,6 & $-16,4$ & 3,3 & $-6,7$ & 7,9 \\
\hline
\end{tabular}

Fuente: EUROSTAT. 


\section{CUADRO II. ESTRUCTURA DE LA POBLACION}

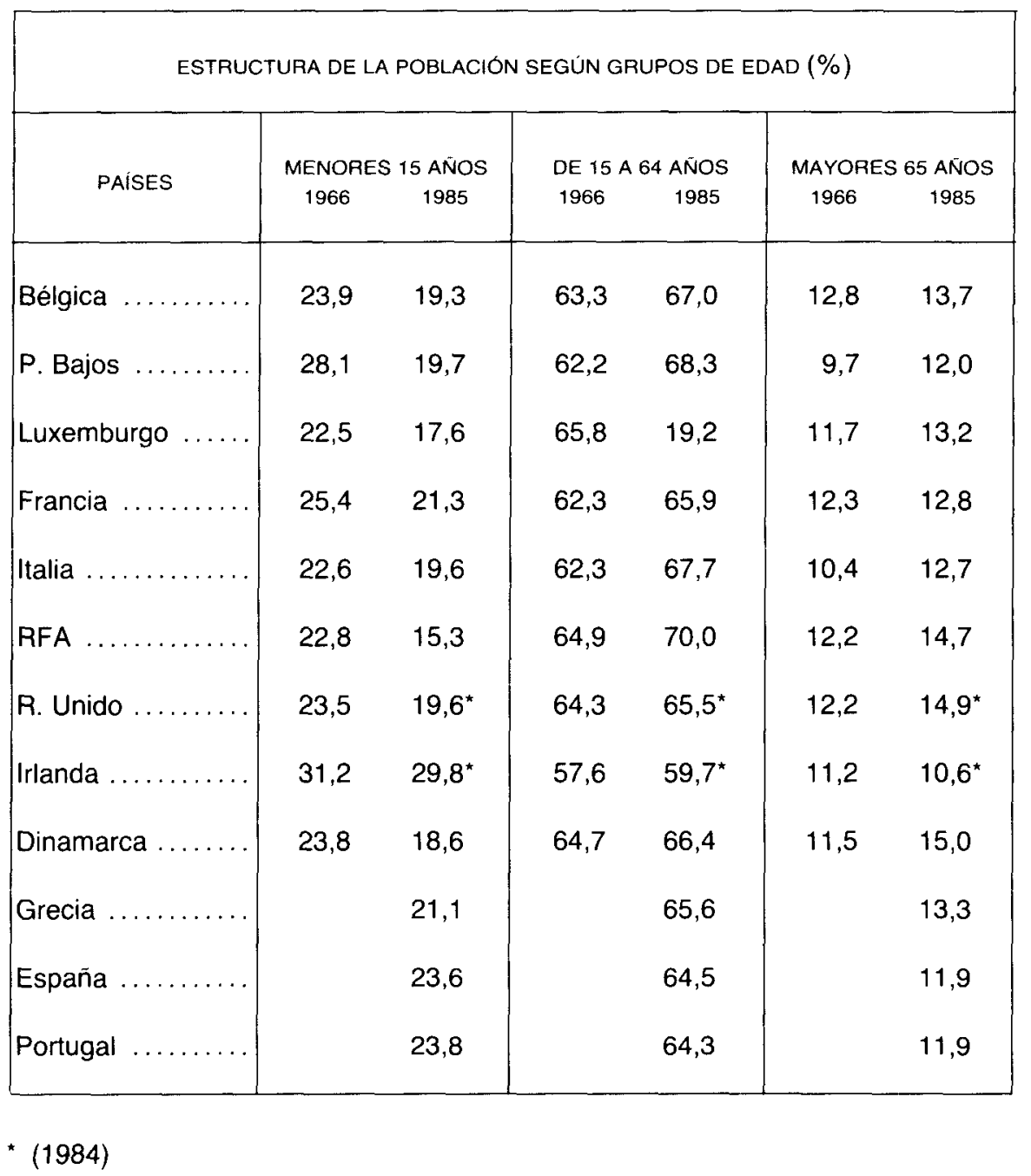

Fuente: EUROSTAT. 
Repercusiones sociales de la entrada de España en la CEE

\begin{tabular}{|c|c|c|c|c|c|c|}
\hline \multicolumn{7}{|c|}{ CUADRO II (Continuación) } \\
\hline \multicolumn{7}{|c|}{ ESTRUCTURA DE LA POBLACION SEGUN GRUPOS DE EDAD (\%) } \\
\hline & \multicolumn{6}{|c|}{ PORCENTAJE DE LA POBLACIÓN EMPLEADA EN: $(\%)$} \\
\hline & SECTOF & GIMARIO & SECTOR & JNDARIO & SECTOI & CIARIO \\
\hline PAISES & 1966 & 1985 & 1966 & 1985 & 1966 & 1985 \\
\hline Bélgica ..... & 5,9 & 3,0 & 46,4 & 30,9 & 47,7 & 66,1 \\
\hline P. Bajos .... & 8,5 & 5,0 & 40,4 & 28,7 & 51,1 & 66,3 \\
\hline $\begin{array}{l}\text { Luxem- } \\
\text { burgo. }\end{array}$ & 11,5 & 5,0 & 46,9 & 38,0 & 41,6 & 57,0 \\
\hline Francia ..... & 17,1 & 8,3 & 40,1 & 34,6 & 42,8 & 57,1 \\
\hline Italia & 25,3 & 12,4 & 37,2 & 37,0 & 37,5 & 50,6 \\
\hline RFA $\ldots \ldots \ldots$ & 10,6 & 5,6 & 48,9 & 42,0 & 40,5 & 52,4 \\
\hline R. Unido ... & 3,7 & 2,7 & 46,4 & 34,4 & 50,0 & 62,9 \\
\hline Irlanda ..... & 31,6 & 17,1 & 27,7 & 29,7 & 40,7 & 53,2 \\
\hline Dinamarca & 13,5 & 7,4 & 37,1 & 28,3 & 49,5 & 64,3 \\
\hline Grecia ..... & & 30,0 & & 28,6 & & 41,4 \\
\hline España .... & & 18,0 & & 33,5 & & 48,5 \\
\hline Portugal .... & & 25,9 & & 37,1 & & 37,0 \\
\hline
\end{tabular}

Fuente: EUROSTAT

Las densidades son muy distintas según las regiones, así, mientras que una cuarta parte del territorio no está ocupado más que por el 5 por 100 de la población, el 20 por 100 de ésta se concentra en una quinta parte del territorio. En líneas generales, estas fuertes densidades quedan asociadas 
a hechos como: actividades económicas intensas (zonas hulleras, zonas industriales, comercio portuario...); zonas de altos niveles de ingresos y de productividad; y la urbanización, de este modo hay regiones que mantienen su crecimiento gracias a la presencia de grandes metrópolis (Dublín, Copenhague...) o a la imbricación de las funciones urbanas (región parisina, Gran Londres, Randstad Holland...). (Gráfico 1.)

Estos hechos globales plantean diversos problemas:

- El elevado volumen actual de mano de obra, que aunque en principio pudiera significar un hecho positivo, dado el contexto económico de crisis que ha atravesado Europa en los últimos años, es una fuente de problemas como lo demuestran los altos índices de paro.

- El constituir un elevado volumen de consumidores, inicialmente implica unas ventajas a nivel económico por las posibilidades que confiere al mercado, aunque este hecho se enturbia en función de la reducción del poder adquisitivo derivado de la crisis económica, que ha llevado, incluso, a unos significativos porcentajes de pobreza.

- El envejecimiento de la población incrementa los costos sociales con las consiguientes repercusiones en materia de seguridad social, cuyas dificultades han llevado a que los distintos Estados tomen medidas aisladas de alcance limitado y poco coordinádas entre sí.

- El descenso de los menores de quince años que preocupa especialmente a la CEE, como se ha manifestado en distintas ocasiones (Conferencia demográfica europea de Strasburgo en agosto de 1971; Consejo de Europa, e incluso los propios Gobiernos), en las que se ha alentado a los Estados a promover políticas natalistas, o se han tomado medidas como: garantizar a las mujeres la conservación del empleo tras el parto con un período de baja postparto variable según los países; mejoras de las concesiones familiares; desarrollo de trabajos a tiempo parcial..., etc.

- Las fluctuaciones podrían entrañar irregularidades en la estructura por edad y tener sensibles repercusiones sobre el plano económico y sobre la sociedad.

- Los contrastes de densidad crean grandes desequilibrios regionales reflejados en hechos tales como: el progresivo empobrecimiento de las regiones más despobladas; costes sociales elevados en las zonas más 
congestionadas; desigualdad en las infraestructuras regionales; diferencias en los empleos especializados y en las posibilidades de rentas que de ello se deriva; desiguales condiciones de vida..., etc.

La política social de la CEE ha prestado especial atención a estos desequilibrios enfocando su acción hacia la financiación de las regiones más pobres; y hacia una descentralización, basando sus objetivos en: la mejora de la calidad del entorno; el alza del nivel de equipamientos regionales; la estabilidad de circuito interregional de la renta; y en la calidad de los equipamientos.

\section{POSIBILIDADES QUE CONFIERE LA POBLACION A LA ESTRUCTURA SOCIOECONOMICA DE LA CEE}

Las posibilidades que confiere esta población a la estructura socioeconórnica de la CEE se derivan de la propia evolución de los movimientos naturales, los cuales han llevado a un crecimiento relativamente rápido de la población activa. La población activa en la CEE representa el $\mathbf{4 4}$ por 100 de la población total, con unas perspectivas de descenso debido al descenso de la natalidad y al descenso de la duración de la actividad, por la prolongación de la escolaridad y la anticipación de la jubilación.

Este crecimiento de la población activa habido hasta el momento, se explica con diversas causas entre las que caben citar: la llegada a la edad activa de la numerosa población nacida antes de 1965; y por la evolución de la mentalidad que ha supuesto el desarrollo del trabajo femenino, que ha pasado de un 35,5 por 100 en 1970 a un 41 por 100 en 1980 , mientras que la población activa masculina sigue prácticamente estacionaria, en torno a un 68,5 por 100 .

Evidentemente, estos hechos junto con la recesión económica de los últimos años, hacen que descienda la demanda de mano de obra extranjera que habia años atrás, tendiendo a reducirse los movimientos de población intracomunitarios.

La evolución de la población activa varía según los Estados Miembros, de forma que podemos agruparlos en: 
- Unos Estados con tasas de actividad elevadas y en crecimiento, entre los que están: Dinamarca $(50,8 \%)$, con un crecimiento del $+1,2$ por 100 al año y una tasa de actividad femenina muy fuerte $(44,4 \%)$; el Reino Unido, con un crecimiento ralentizado del +3 por 100 al año; Francia, Bélgica y Luxemburgo.

- Otro grupo de Estados con tasas de actividad en disminución, entre los que se encuentran: la RFA, como consecuencia de la caída precoz de la natalidad y de la partida, después de 1975, de numerosos trabajadores extranjeros; Irlanda, donde la tasa de actividad es la más baja de CEE $(33,7 \%)$, en relación con el elevado porcentaje de jóvenes en la población total, y de una tasa de actividad femenina muy débil (18\%); y Grecia, Italia y los $\mathrm{P}$. Bajos, con tasas relativamente débiles pero en alza.

Las variaciones del empleo femenino han jugado un importante papel en la evolución de la población activa. En nuestro país, la mano de obra femenina se caracterizó por su flexibilidad y por su sensibilización a las situaciones de demanda; hay quien ha llegado a senalar que ha tenido un papel de "ejército de reserva" habiendo estado en relación con las necesidades de mano de obra requeridas por el sistema económico.

Si miramos hacia atrás, vemos que la expansión económica de los años 60 introdujo variaciones fundamentales en la composición de la población activa, que afectó de forma más acusada a la mano de obra femenina. Así se comenta que la mujer comenzó realmente a incorporarse al mundo laboral cuando la expansión económica agotó los efectivos masculinos y se produjo una ampliación de empleos disponibles en el sector terciario. Entre 1964 y 1980 el indice de actividad femenina aumentó notablemente, apreciándose una marcada inflexión con la crisis económica de los años 70.

En los últimos años se aprecian ya otras consideraciones con respecto a la incorporación de la mujer al trabajo, al tiempo que se produce un comportamiento cada vez más autónomo de la oferta de trabajo femenino como consecuencia de: una mayor resistencia de la población femenina a abandonar su actividad, debido a un cambio de mentalidad sobre su papel en la sociedad y a un aumento de su nivel educativo; a la incorporación de nuevos sectores de mujeres al mercado laboral, inducidos por la disminución de recursos económicos en su núcleo familiar; y como consecuencia de los cambios en la demanda empresarial, con requerimiento de mano de obra femenina hacia sectores concretos del mercado. 
Según cifras de 1984, las mujeres representaban el 26 por 100 del total de la población activa, aunque todavía un 17 por 100 tenia la consideración de ayuda familiar. La TAF presentaba patentes diferencias según la edad, el estado civil y la cualificación profesional:

ACTIVIDAD SEGÚN EL NIVEL DE ESTUDIOS

Casadas $\% \quad$ Solteras \%

Mujeres con estudios primarios

18

62,3

Mujeres con estudios superiores

72,8

81,3

ACTIVIDAD SEGÚN LA EDAD

Mujeres entre 20-24 años: $57 \%$

Mujeres entre 25-29 años: 5 de cada 10 mujeres.

Mujeres entre 30-39 años: 3 de cada 10 mujeres.

No obstante nuestra TAF del 26 por 100 , queda por debajo de la media Europea que se encuentra en el 38 por 100.

El volumen de la población activa tiene una gran importancia en cuanto al exceso o demanda que presentarán los países de la CEE, por lo que es interesante preguntarnos sobre qué porcentaje representa la mano de obra extranjera dentro de la población activa comunitaria. Tradicionalmente la mano de obra extranjera ha representado un porcentaje importante sobre todo en RFA, Francia y el Reino Unido: en la RFA los trabajadores extranjeros compensaban la insuficiente mano de obra nacional, representando el 7,8 por 100 de la población activa, repartidos por orden de mayor a menor entre turcos, yugoslavos, italianos y griegos. En Francia representaban el 7,4 por 100 de la población activa, repartidos entre portugueses, argelinos, españoles, marroquies e italianos. Y en el Reino Unido se encontraban numerosos trabajadores de color originarios de la Commonwealth, como antillanos, pakistanies, o indios, que se beneficiaban de los mismos derechos que los ciudadanos británicos. 
En los últimos años estos porcentajes han decrecido, pero, a pesar del incremento de la población activa autóctona y del elevado porcentaje de paro, todavía se aprecia en algunos paises un porcentaje importante de población inmigrante: 26,2 por 100 en Luxemburgo, 9,0 por 100 en Bélgica, 7,4 por 100 en Alemania, 6,7 por 100 en Francia, 6,6 por 100 en Irlanda, 3,8 por 100 en Holanda y Reino Unido, 2,6 por 100 en Grecia, 2 por 100 en Dinamarca y entre 0,8 y 0,6 por 100 respectivamente, en Italia, España y Portugal.

En este sentido es importante la comparación de los convenios de inmigración, pues con anterioridad a nuestra adhesión a la CEE, países del Tercer Mundo gozaban de mayores ventajas que los españoles.

El paro es un problema que, al tiempo que plantea importantes problemas socioeconómicos, dificulta la movilidad intracomunitaria, siendo todavia preocupante la situación en 1986. En este año se apreciaba:

- Un alto nivel de desempleo, aunque también hay que decir que en este año había aumentado el volumen de empleo, no obstante la lucha contra el paro sigue siendo uno de los principales objetivos sociales. Solamente habian reducido el desempleo Alemania, Dinamarca y Benelux.

- Un ligero aumento del número de demandantes de empleo.

- Un incremento del porcentaje de desempleados de larga duración, en su mayor parte se trata de trabajadores poco o nada cualificados y por lo tanto difíciles de contratar. Concretamente en otoño de 1986, un 40 por 100 de los desempleados llevaban más de un año sin trabajo; siendo este porcentaje superior al 50 por 100 en algunos paises.

- Los menores de 25 años seguían constituyendo una parte importante de los desempleados (38\% del total), variando su importancia según los países: entre el 44 y 47 por 100 en Italia, España y Luxemburgo, más del 35 por 100 en Francia, Bélgica, P. Bajos y Reino Unido, e inferior al 25 por 100 en la RFA y Dinamarca.

La acción de la politica comunitaria se orienta hacia la incorporación laboral de esta población mediante la formación profesional en nuevas tecnologias o mediante el fomento de iniciativas tendentes a la creación de puestos de trabajo. 
El gráfico 2 muestra la relación entre población activa y paro según datos estadísticos de EUROSTAT en 1984.

\section{Estructura de la población activa}

En 1984, la Europa de los diez presentaba una estructura muy avanzada: se observaba un peso muy reducido de los activos primarios, del 7 por 100 y en descenso; un potente sector secundario, que representaba el 34 por 100 de la población activa, y que experimentaba una ligera disminución del - $1 \%$ /año; y un sector terciario en continuo crecimiento, en torno al $+1,8 \%$ /año, y que en ese momento ocupaba al 59 por 100 de la población activa. Dentro de este sector terciario, los países que han experimentado un mayor alza han sido: Grecia, Dinamarca, Italia y Luxemburgo.

Esta situación se ha alterado ligeramente con el ingreso de España y Portugal que tienen un mayor peso en el sector primario.

Individualizadamente, existe una gran diferencia entre los distintos países de la Comunidad con valores que oscilaban en 1983: entre el 28,5 por 100 de Grecia y el 2,6 por 100 del Reino Unido para el sector primario; entre el 41,1 por 100 de la RFA y el 27,2 por 100 de Grecia para el sector secundario; y entre el 67,8 por 100 de los Países Bajos y el 40 por 100 de Portugal para el sector terciario. (Cuadro II).

En este sentido habria que puntualizar un hecho, y es que las disparidades regionales son aún más marcadas que las nacionales.

Las repercusiones que tendrá la adehesión de España a la CEE sobre nuestra población activa son muy numerosas y entre ellas hemos de destacar las siguientes:

- Las estructuras de empleo, que deberán experimentar una reestructuración sectorial.

- La movilidad interocupacional, debida a un mercado de trabajo mucho más amplio y ante el cual es básico dedicar una fuerte atención a la formación profesional y a la orientación de los movimientos migratorios, en función de las necesidades detectadas y de los planes previstos de reestructuración sectorial y de desarrollo regional. 
- Las condiciones de trabajo y derechos laborales, al acomodarse nuestra legislación a la legislación comunitaria y al irse aplicando distintas mejoras ya obtenidas por los trabajadores de otros países de la CEE, como pueden ser: la reducción de la jornada de trabajo, el aumento del período de vacaciones, ciertas prestaciones de asistencia social..., etc.

- La promoción de la igualdad de oportunidades y de participación en la vida económica y social para hombres y mujeres, en lo que España está muy por debajo de los países europeos tanto a nivel legislativo como a nivel de la vida contidiana. En este sentido, el segundo programa de acción comunitaria para 1986-90 tiene por objeto un importante número de acciones referentes al empleo de las mujeres, para favorecer principalmente una igual participación en los empleos relacionados con las nuevas tecnologías. Por otra parte, la Comisión propone acciones en favor de un reparto más equitativo de las responsabilidades profesionales, familiares y sociales, debiéndose dar una especial importancia a las campañas de información y sensibilización, tanto del público en general como de ciertos medios profesionales. Al mismo tiempo se establece también la necesidad de una concertación intensiva entre las autoridades comunitarias, nacionales y regionales.

- Fomento del empleo para paliar el acuciante problema del paro que ha sufrido la población europea con la recesión económica de los últimos años. La resoluciones tomadas por la CEE en este sentido van encaminadas a promover las iniciativas locales; a fomentar los proyectos innovadores; a promover la fundación de empresas con la consiguiente creación de puestos de trabajo; a conseguir un mejor funcionamiento del mercado de trabajo; a aumentar las posibilidades de formación de jóvenes y adultos; a ampliar la cooperación de los Estados Miembros, a aumentar las ayudas a los desempleados de larga duración..., etc., y a la creación de grupos de trabajo, como por ejemplo el de Val Duchesse en 1985, donde la Comisión creó dos grupos: el de "macroeconomía", para ocuparse de la aplicación práctica de la estrategia conjunta de crecimiento a fin de incrementar el empleo, y el de "nuevas tecnologías y diálogo social», para examinar los diversos aspectos ligados a la introducción de nuevas tecnologías en el mercado laboral.

Este aspecto, a pesar de todas las iniciativas mencionadas, sigue resultando un grave problema a nivel general y no parece tener una solución inmediata como vimos al analizar la población en paro dentro de la CEE en 1986. 
- Las condiciones de movilidad de los trabajadores dentro de la CEE como veremos a continuación al analizar los movimientos migratorios.

\section{Los movimientos migratorios}

La libre circulación de trabajadores constituye uno de los principales puntos en los que se centra la política social comunitaria.

Dentro de la CEE debemos diferenciar paises con salida de trabajadores y países de acogida o de inmigración. Entre los países de carácter emigratorio se encuentran Italia, España, Irlanda, Grecia, Portugal y Gran Bretaña; y entre los países de inmigración están la RFA, Holanda, Francia y Bélgica.

Estas migraciones, al no ser tradicionalmente definitivas y familiares sino temporales y de mano de obra, no han tenido todas las consecuencias demográficas que cabría esperar de su magnitud. No obstante, la importancia de la emigración en cada época refleja las vicisitudes de la coyuntura económica: movimientos importantes hasta 1973 debido al fuerte crecimiento económico habido desde los años 50 ; saldos migratorios negativos tras la recesión económica de los años 70 y distintas oscilaciones en los últimos años.

El problema del paro ha limitado notablemente los movimientos migratorios por motivos laborales, a través de las medidas tomadas por muchos países para restringir la llegada de trabajadores de otros paises, o para incitar a los trabajadores extranjeros a volver a sus países. Evidentemente, en estos casos la situación es distinta para los trabajadores comunitarios que para los que no pertenecen a la CEE, ya que los reglamentos de la CEE que aseguran la libertad de circulación y de instalación de trabajadores, acuerdan una prioridad al mercado de trabajo comunitario. No obstante estos acuerdos quedan un tanto en entredicho, no sólo porque la oferta y la demanda de empleo puedan variar, sino porque la equiparación entre los ritmos de desarrollo entrañan una disminución de los desplazamientos intracomunitarios. Por otro lado se favorece a través de acuerdos bilaterales, la entrada de trabajadores poco cualificados del Tercer Mundo, que ofrecen mano de obra barata, aceptan tareas más ingratas y están menos politizados.

En este sentido, el documento de negociación español para la adhesión a la CEE consideró que los acuerdos suscritos por la CEE con terceros países suponían en determinados casos discriminaciones para nuestros tra- 
bajadores, solicitándose al menos una equiparación con ellos a partir de la adhesión. Ello significa, pues, una ventaja de entrada.

Cuando nos planteamos las repercusiones de nuestra entrada en la CEE desde el punto de vista de las migraciones laborales, tendemos a interesarnos por qué derechos, libertades y posibilidades de trabajo tendrán nuestros emigrantes; ahora bien, debemos tener muy en cuenta que también habrá una contrapartida, es decir, que el movimiento laboral se efectuará en ambos sentidos.

En lo que respecta a nuestros emigrantes, hay que señalar que actualmente hay en los Estados Miembros de la CEE alrededor de unos 240.000 trabajadores españoles con 366.000 familiares a su cargo directo, de los cuales hay unos 25.000 en paro. Desde la adhesión, todos ellos tienen derecho al mismo trato que los trabajadores comunitarios en lo referente a materias socio-laborales, fiscales, sindicales, de acceso a la vivienda, transporte y escolarización de los hijos; y los emigrantes ya instalados que tengan miembros de su familia residiendo en España recibirán las prestaciones familiares del país de empleo tras un período de tres años.

En cuanto a la libertad de circulación, se establece un período transitorio de siete años (excepto en el caso de Luxemburgo, donde se aplicarán 10 años) con una cláusula de revisión a los cinco años.

Por su parte, la incorporación de los países comunitarios a nuestro mercado de trabajo deberá llevar consigo una modificación de las disposiciones españolas en materia de inmigración. Hasta ahora, las disposiciones españolas se basaban en la limitación de las concesiones de residencia a los trabajadores extranjeros, supeditándolas a razones económicas y dando prioridad total a los trabajadores españoles sobre los extranjeros. Ahora, en cambio, deberá permitir la entrada a los beneficiarios de la CEE con la simple presentación de la tarjeta de identidad.

De este modo, si no se toman medidas referentes a la preparación profesional de nuestra mano de obra, la posibilidad de libre circulación de trabajadores en doble sentido, unido al deterioro de las posibilidades de empleo comunitarias, puede provocar una invasión de mano de obra en nuestro país. En este sentido preocupa sobre todo el sector servicios y en especial las ocupaciones relacionadas de alguna forma con los turistas y los residentes extranjeros; ello sería especialmente grave si consideramos que estas ocupaciones afectarian a las zonas menos desarrolladas del país. 
Los rasgos generales de las caracteristicas y de la situación de la población emigrante nos hará comprender mejor las repercusiones que tendrá la política comunitaria sobre la emigración española. En los últimos años se han apreciado algunos cambios siendo de destacar los siguientes aspectos:

- El cambio en la dirección de las migraciones, orientándose además de hacia los paises tradicionales de acogida, hacia otros países como Reino Unido, Suiza y Holanda.

- Tendencia hacia la emigración de carácter familiar en oposición a la emigración individual tradicional.

- Elevación del nivel de estudios del emigrante, incrementándose el porcentaje de técnicos y estudiantes que son menos sensibles a las oscilaciones de la coyuntura económica. De entre ellos, la población más cualificada se dirige al Reino Unido, y la menos cualificada hacia Suiza (servicios y construcción).

- El perfil ocupacional predominante se sigue situando en trabajos industriales y de servicios sin una especial cualificación, y sólo un pequeño porcentaje se emplea en funciones técnicas, medias o superiores. La distribución por sectores de actividad varía según los países: en Francia predominan los trabajadores españoles dedicados al servicio doméstico y la construcción; en Alemania y Países Bajos los dedicados a la industria; en Suiza los dedicados a la construcción y a la hostelería, y en el Reino Unido a la hostelería.

- Los problemas de integración, debido al incremento de la hostilidad que se ha apreciado en los últimos años, al ser considerados los inmigrantes como competidores en los momentos de crisis económica. Ante este hecho hubo de hacerse el 11-6-85 una declaración conjunta del Parlamento, el Consejo y la Comisión contra el "racismo y la xenofobia". Las cifras de la población emigrante española indican que un 24 por 100 de la población se declara no integrada, integración que varía en función de la edad, el conocimiento del idioma, los amigos, el nivel cultural, el tiempo de permanencia... etc. Analizado por países, también varía el nivel de integración, así, los paises en que la población española se siente más integrada son Reino Unido, Bélgica y Francia, en cambio se considera más discriminada en Suiza, RFA y Holanda. 
En esta integración que se propone la CEE debe jugar un importante papel la política estatal, debiendo promocionar e impulsar la integración social y cultural.

\section{ASPECTO SOCIOCULTURALES}

Las características socioculturales no son tan desequilibradas entre los paises componentes de la CEE como en el caso de otras características poblacionales sino que parecen más homogéneas. Observando el conjunto comunitario y relacionándolo con la población mundial, puede decirse que es la población con menor tasa de analfabetismo, con niveles educativos más generalizados, largos y avanzados, y con mayor riqueza y tradición cultural, aunque queda en muchos de ellos a gran distancia de la población USA.

No obstante, la lucha contra el analfabetismo es todavia una acción que trata la política social, puesto que ningún pais se salva de él, a pesar de que en los años 60 y 70 se creyó que el alza general del nivel de escolarización bastaría para combatirlo, pero no ha sido así. Por ello se planteó en 1984 una coordinación a nivel europeo para mejorar los sistemas de educación y realizar programas de alfabetización de adultos.

Dentro de la política educativa comunitaria hay otros puntos importantes: así, el libre acceso a la educación se considera como derivado de la libre circulación de personas, previéndose que los habitantes de la CEE deberán poder estudiar libremente en el lugar de su elección.

Otros problemas se plantean en cuanto a la equivalencia de los diplomas y en cuanto al reconocimiento de las cualificaciones profesionales y de estudios.

Si analizamos la situación española en cuanto al grado de desarrollo educativo en relación con la CEE, veremos que resulta más o menos equiparable según los aspectos que observemos. Si nos basamos en un estudio realizado por la Secretaría General Técnica del Ministerio de Educación y Ciencia en 1981, podríamos considerar que nos equiparamos en cuanto a la organización del sistema educativo; en cuanto a las exigencias de esco- 
larización en los diversos niveles; y en cuanto a las tasas de escolarización, si bien éstas son algo inferiores en los niveles medios.

Por el contrario, presentamos una evidente inferioridad en la asignación de recursos al sistema educativo público, tanto en términos de valores por habitante, por alumno oficial, o en términos de porcentaje del Producto Nacional Bruto. Igualmente la calidad de la enseñanza en España está en inferioridad con respecto a los paises de la Comunidad.

Es decir, que si en el orden cuantitativo no existen diferencias realmente apreciables, la menor cantidad relativa de los medios económicos dedicados por España sí repercuten en la calidad.

Un aspecto importante es la repercusión que podrá tener nuestra participación en proyectos educativos comunitarios, de los que posiblemente obtendremos notables beneficios. Son de considerar entre ellos: el proyecto COMETT dedicado a fomentar la realización de prácticas en empresas de otros Estados Miembros, y programas de formación conjunta para obtener especialistas cuyos conocimientos respondan al rápido progreso técnico; y los programas de intercambio universitario ERASMUS, YES, COMETT y de formación profesional EUROTECNET.

\section{ALGUNAS REPERCUSIONES APRECIADAS TRAS LA ADHESION}

Dos años después de nuestra entrada en la CEE, existen pocos balances concretos sobre las repercusiones habidas hasta el momento, por lo que a título de ejemplo mencionaremos el realizado por la CAM (Comunidad Autónoma de Madrid). En él se considera que el inicio de la relación CEECAM ha producido diversas influencias, algunas de las cuales han resultado inesperadas. De este balance, en el aspecto social podemos destacar los siguientes puntos:

- Se han producido los primeros flujos financieros procedentes de los fondos comunitarios, es decir, del Fondo Social para ayudas al empleo y a la formación profesional, que cubrieron el 50 por 100 del importe de los programas realizados en la CAM, y del que se beneficiaron 50.312 trabajadores, 
siendo los más favorecidos los menores de veinticinco años en lo que concierne a la formación profesional. Para el año 1987 las previsiones de la inversión eran aún mayores, de más del doble del año anterior, pensándose que el número de personas beneficiadas ascendería a unas 105.500.

- Se han creado diversos organismos de coordinación en materias relacionadas con la CEE, entre los que caben señalar el Patronato Madrileño de Asuntos Europeos que constituye una de esas entidades intermedias, tan propiciadas por la CEE, destinada a reforzar el tejido social comunitario mediante la divulgación, la formación y la documentación de temas relacionados con la CEE.

- En el área de consumo se comenzaron a ver las inadecuaciones de nuestro ordenamiento interno al comunitario, dando lugar a la realización de normas más o menos rigurosas según los casos, y realizando campañas de educación del consumidor según las líneas dadas por el Consejo de Ministros de Educación de la CEE el 9-6-86.

- Se inició la labor de adaptación de las normativas medioambientales a la legislación comunitaria, referentes a la protección general de los valores medioambientales, desarrollo de un programa coordinado de actuación sobre residuos industriales, plan de saneamiento atmosférico de la CAM, y formación de personal técnico destinado a los problemas ambientales.

A esta política del medioambiente se le quiere dar una dimensión tal, que llegue a ser un componente esencial de toda la política económica y social comunitarias, ya que entre sus objetivos están: garantizar una buena gestión de los recursos naturales; introducir aspectos cualitativos en la planificación y organización del desarrollo social y económico; tomar medidas ambientales precautorias, y prever costes de planificación e investigación. Además, se piensa que, socialmente, una política del medioambiente consolidada, tendrá efectos positivos en la creación de empleos mediante la creación de una importante infraestructura ambiental y el estímulo de las inversiones, así como a través de la fabricación de nuevos productos que estén directamente relacionados con la mejora en la calidad del medioambiente. 


\section{BIBLIOGRAFIA}

Comision de las Comunidades EuRopeas (1985): Orientaciones para una política comunitaria de las migraciones. Bruselas.

Comision de las Comunidades EuRopeas (1987): Mujeres de Europa. Bruselas. 43 págs.

COMMISSION DES COMMUNAUTÉSEUROPÉNNES (1977): La concentration geographique dans les pays de la CE. Bruxelles.

COMUNIDAD EUROPEA (1984): “El presupuesto de la Comunidad Europea". Serie Documentos, núm. 15. Madrid. 11 págs.

—: (1986) «La política social de la Comunidad Europea». Serie Documentos.

—: (1986) «Hacia la Europa de los ciudadanos». Serie Documentos núm. 2.

—: (1985) «España y la Comunidad». Serie Documentos núm. 8.

-: (1987) «Los trabajadores españoles y portugueses en la Comunidad europea". Informaciones sindicales. Bruselas. 35 págs.

CONSEIL DE L'EUROPE (1978): Seminaire sur les incidences d'une population stationaire ou decroissante en Europa. Ed. Orduña. Liege. 349 págs.

DOMingUEZ, J. C. (Coordinador) (1985): Emigración y mercado común. Univ. Intern. Menéndez Pelayo. Inst. Esp. de Emigración. Santander. 
GOMEZ DE ENTERRIA Y SÁNCHEZ, C. (1984): «La aplicación en España de la libertad de establecimiento y la libre prestación de servicios de las personas físicas". Documentación Administrativa, núm. 201. Madrid, págs. 413-446.

Haering Pérez, F. (1984): «La acción de la Comunidad Económica Europea en materia de convalidación de títulos y diplomas". Documentación Administrativa, núm. 201. Madrid, págs. 447472.

MigueL, A. de (1986): “Panorama de la emigración española en Europa". Centro de publicaciones del Ministerio de Trabajo y Seguridad Social. Madrid.

Ministerio de EdUCACIÓN Y CIENCIA (1981): La educación en España y en la CEE. Estudios de Educación. Secretaría General Técnica. Madrid.

Peredo Linacero, J. A. (1984): “Los aspectos sociales de la integración en la Comunidad Económica Europea: sus repercusiones en España". Documentación Administrativa, núm. 201, Madrid, págs. 353-412. 
Repercusiones sociales de la entrada de España en la CEE

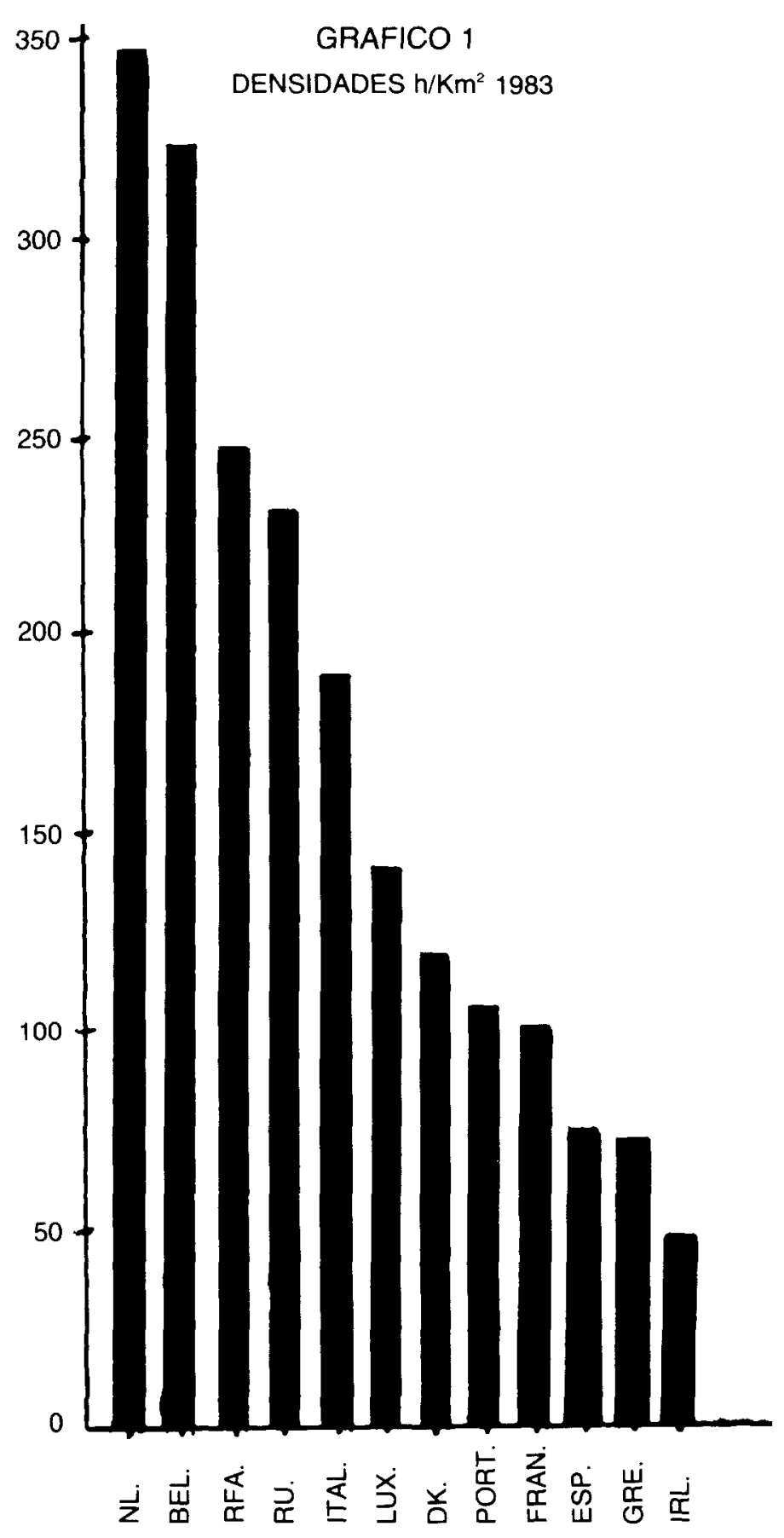




\section{GRAFICO 2}

POBLACION ACTIVA (\%) 1984
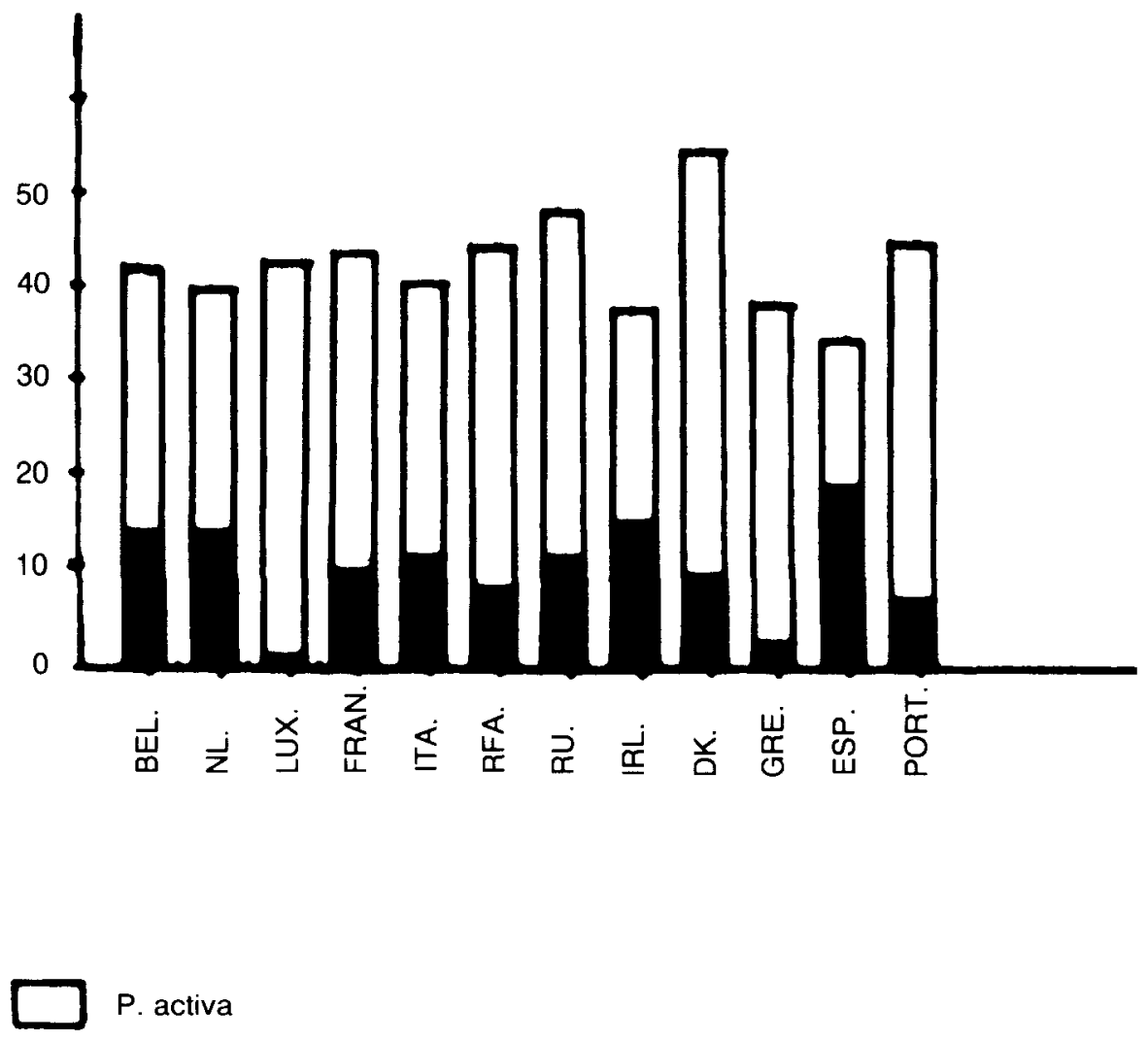

P. activa

$P$. en paro 